DOI: 10.12957/demetra.2016.18460

\title{
Desenvolvimento de questionário de frequência alimentar para população adulta residente em Santo Amaro, Bahia, Brasil
}

\author{
Development of food frequency questionnaire for adult population living in Santo Amaro, \\ Bahia, Brazil
}

\author{
Valéria Macedo Almeida Camilo' \\ Fernanda de Freitas Virgínio Nunes \\ Darcilene Fiuza da Silva' \\ Isabella de Matos Mendes da Silva' \\ Fábio Santos de Oliveira' \\ Jerusa da Mota Santana ${ }^{2}$

\begin{abstract}
1 Universidade Federal do Recôncavo da Bahia, Centro de Ciências da Saúde. Santo Antônio de Jesus-BA, Brasil.

2 Universidade Federal da Bahia, Instituto de Saúde Coletiva, Programa de Pós-graduação em
\end{abstract} \\ Saúde Pública. Salvador-BA, Brasil.
}

Correspondência / Correspondence Valéria Macedo Almeida Camilo E-mails:valeria_m@terra.com.br , vcamilo@ufrb.edu.br

\section{Resumo}

O objetivo do estudo foi descrever a elaboração de um questionário de frequência alimentar para população adulta residente de Santo Amaro, Bahia, Brasil. Trata-se de estudo transversal conduzido com 50 indivíduos adultos de ambos os sexos, no município de Santo Amaro no período de agosto a setembro de 2013. A relação foi composta por 91 itens alimentares distribuídos em sete categorias de consumo e em 15 grupos alimentares. O QFA inicial foi aplicado na população estudada, juntamente com o R24h. Do QFA inicial foram excluídos 38 produtos alimenticios, o que representa $41,77 \%$ da lista. Os alimentos citados por no mínimo $15 \%$ da população estudada foram mantidos no QFA, com destaque para o grupo das frutas, que apresentou percentual de consumo maior ou igual a 90\%; grupo das hortícolas, grupo dos açúcares e açucarados; laticínios; produtos de panificação; bebidas; carnes; grãos; massas e farináceos. A lista final do QFA apresentou 53 itens alimentares. Destaca-se que, com esse instrumento, a avaliação do consumo alimentar terá maior fidedignidade, por se aproximar da realidade local da população em estudo, contribuindo com as pesquisas que investigam a relação entre consumo alimentar e condições de saúde. Concluise que o QFA inicial, assim como o R24h, foram instrumento importantes que delinearam a construção do QFA proposto, e que este levou em consideração o hábito alimentar da população estudada, expressando maior qualidade ao instrumento, praticidade e otimização do tempo de aplicação.

Palavras-chave: Consumo de Alimentos; Questionário; Comportamento Alimentar; Epidemiologia. 


\section{Abstract}

This study aimed to describe the elaboration of a food frequency questionnaire (FFQ) for the adult population living in Santo Amaro, Bahia, Brazil. It was a cross-sectional study conducted with 50 adult subjects of both sex, in the city of Santo Amaro from August to September, 2013. The roll of items was composed of 91 food items distributed in 7 categories of consumption and in 15 food groups. The first FFQ was applied, on the study population, with the $24 \mathrm{~h}$ recall $(\mathrm{R} 24 \mathrm{H})$. From the initial FFQ, 38 food products were excluded, representing $41.77 \%$ of the list. The food mentioned by at least $15 \%$ of the study population were kept in the FFQ, highlighting the group of fruits, with a consumption percentage larger than or equal to $90 \%$; of the groups of vegetables, sugars and sugary, dairy, bakery products, beverages, meats, grains, dough and farinaceous. The final FFQ list presented 53 food items. Noteworthy, with this instrument, food consumption assessment will be more reliable, as it approximates to the local reality of the studied population, contributing with researches that investigate the relationship between food consumption and health conditions. The conclusion is that the initial FFQ associated with the R24h were important instruments that outlined the construction of the proposed FFQ, which considered the food habit of the study population, expressing higher quality to the instrument, practicity and optimized application time.

Key words: Food Consumption; Questionnaires; Feeding Behavior; Epidemiology.

\section{Introdução}

A epidemiologia nutricional tem evidenciado que o padrão alimentar da população brasileira encontra-se em inversão nas últimas duas décadas. Constata-se que o consumo de alimentos naturais, como frutas, verduras, legumes, leguminosas e raízes, tem sido substituído por alimentos fontes em açúcares, gorduras saturadas e trans, alimentos industrializados e/ou ultraprocessados. ${ }^{1}$ Esse padrão alimentar identificado ao longo do tempo, associado a mudanças no estilo de vida, caracteriza a transição nutricional e tem sido identificado na literatura científica como fator de risco modificável para ocorrência de doenças crônicas não transmissíveis em todos os ciclos da vida. ${ }^{2,3}$ 
Para que estes padrões possam ser investigados, é fundamental analisar o consumo alimentar dos indivíduos ou da população por meio de inquéritos de consumo alimentar. Dentre os mais utilizados na epidemiologia nutricional, destaca-se o Questionário de Frequência Alimentar (QFA). ${ }^{2,45}$ Este é descrito na literatura científica como instrumento capaz de classificar os indivíduos de acordo com seus padrões alimentares habituais e associá-los às condições de saúde. Salientase que é um instrumento de fácil aplicabilidade e baixo custo, o que viabiliza sua utilização em estudos populacionais. ${ }^{6}$

Esse método de inquérito dietético é elaborado para obter informações qualitativas ou quantitativas sobre a ingestão de alimentos ou nutrientes específicos, ${ }^{7}$ sendo também empregado para investigar o consumo alimentar usual em períodos de curto ou longo prazo. ${ }^{8,9}$

O consumo alimentar da população brasileira imprime diferenças regionais e é influenciado e condicionado por diversos fatores, a exemplo da cultura, condições socioeconômicas, disponibilidade de alimentos, acesso ao alimento, condições fisiológicas e necessidades nutricionais inerentes de cada fase de vida humana. ${ }^{10}$ Assim, segundo Willet $^{6}$ e Cade et al., ${ }^{11}$ para elaborar um instrumento de identificação do consumo alimentar, deve-se considerar todos esses fatores, além dos objetivos da pesquisa, o desenho do estudo, os recursos disponíveis e as características singulares da populaçãoalvo.

Diante do exposto, fica evidente a necessidade de desenvolver um instrumento para avaliação do consumo alimentar por região, uma vez que cada população apresenta particularidades específicas que devem ser consideradas com a finalidade de se aproximar da realidade alimentar do grupo estudado. Nesse sentido, o presente estudo teve como objetivo descrever a elaboração de um questionário de frequência alimentar para população adulta residente em Santo Amaro, Bahia, Brasil.

\section{Metodologia}

\section{População de estudo}

Trata-se de estudo transversal conduzido com indivíduos adultos de ambos os sexos, usuários do Sistema Único de Saúde (SUS) do município de Santo Amaro, Bahia, Brasil, no período de agosto a setembro de 2013. Foram considerados elegíveis para participar do estudo, usuários cadastrados nas Unidades de Saúde da família (USF), com idade maior ou igual a 18 anos residentes e domiciliados na cidade onde foi desenvolvido o estudo; e como inelegíveis, adolescentes e adultos com problemas de saúde. Salienta-se que não houve recusas. 
O estudo constitui-se de amostra por conveniência, em que se adotou o mínimo amostral necessário para elaboração e validação de instrumento para avaliação dietética, conforme sugerem Burley $^{12}$ e Slater et al. ${ }^{9}$ Assim, o estudo apresenta 50 indivíduos, constituintes da primeira etapa do estudo-matriz, que teve como objetivo identificar os hábitos alimentares da população, e assim desenvolver instrumento plausível para a realidade alimentar local.

$\mathrm{Na}$ abordagem inicial do estudo, foram apresentados os propósitos da pesquisa, e aqueles que aceitaram participar responderam a um questionário com informações socioeconômicas e demográficas; foi aplicado um QFA inicial, juntamente com Recordatório de 24h (R24h). Os participantes da pesquisa assinaram um Termo de Consentimento Livre e Esclarecido (TCLE), conforme preconiza a Resolução no 466/2012 do Conselho Nacional de Saúde, ${ }^{13}$ ficando livres em optar por não participar da pesquisa quando acreditassem ter sua integridade física e moral atingidas. O projeto-matriz, que inclui a subamostra do estudo, foi aprovado pelo Comitê de Ética da Universidade Federal do Recôncavo da Bahia, em março de 2013 (CAAE: 04022312.0.0000.0056 e parecer: 219.566).

A coleta de dados foi realizada no período de agosto a setembro de 2013, na cidade de Santo Amaro, Recôncavo da Bahia, Brasil.

\section{Construção do QFA inicial}

O QFA utilizado foi construído com base nos dados obtidos pelo Instituto Brasileiro de Geografia e Estatística (IBGE), ${ }^{14}$ Censo 2006, onde consta a produção agrícola municipal na cidade de Santo Amaro-BA, e nas Pesquisas de Orçamentos Familiares(POF) ${ }^{14}$ 2008/2009. Visou-se obter dados mais fidedignos sobre os produtos produzidos pela região e o orçamento das famílias e hábitos de consumo, com base na literatura sobre alimentos regionais característicos da população do nordeste do Brasil. ${ }^{15}$

A relação foi composta por 91 itens alimentares distribuídos em sete categorias de consumo (nunca; menos de uma vez ao mês; de uma a três vezes ao mês; uma vez por semana; de duas a quatro vezes por semana; uma vez ao dia; e duas ou mais vezes ao dia) e distribuídos em 15 grupos alimentares. Consideraram-se a similaridade das características nutricionais dos alimentos e os aspectos culturais e regionais, com base na pirâmide alimentar brasileira.

\section{O projeto-piloto e a reestruturação do QFA}

Foi selecionada uma amostra mínima para o teste-piloto do estudo maior, intitulado "Avaliação de riscos no consumo de alimentos potencialmente contaminados por metais pesados em Santo 
Amaro-BA". Nesse piloto, foram testados o questionário geral da pesquisa, o QFA inicial construído com base nos alimentos citados na POF 2003/2009, ${ }^{16}$ bem como os alimentos regionais. Foi então aplicado o R24h, inquérito alimentar necessário para conhecer os alimentos mais consumidos da população adulta residente do município. Esse instrumento forneceu subsídios para inclusão dos alimentos habituais na lista do QFA a ser elaborado. Enfatiza-se que o R24h atua como padrão de referência para comparar os dados investigados pelo QFA em estudos de validação e calibração. ${ }^{9}$

Assim, os participantes responderam ao QFA inicial e o R24h em 40 minutos. Para aplicação do QFA, o pesquisador apresentou ao participante uma lista de alimentos e bebidas e solicitou a frequência de consumo, se diário, semanal, mensal ou nunca (nunca; menos de uma vez ao mês; de uma a três vezes ao mês; uma vez por semana; de duas a quatro vezes por semana; uma vez ao dia; e duas ou mais vezes ao dia). Quanto ao R24h, solicitou-se ao entrevistado que relatasse o consumo de todos os alimentos e bebidas ingeridos num período de 24 horas. Empregaramse também técnicas de recordação, como a listagem rápida, descrição detalhada e revisão. Essa técnica contribui com a memória do entrevistado.

As entrevistas foram realizadas por discentes dos cursos de Nutrição e Ciências Biológicas, previamente treinados pela equipe de pesquisadores do projeto. Após aplicação e revisão dos instrumentos, realizou-se a comparação entre os alimentos referenciados no R24h e os alimentos inseridos na lista do QFA inicial. Para que o alimento citado no R24h fizesse parte da lista do QFA, adotou-se como critério a frequência de consumo de pelo menos uma vez por semana, caracterizando-o, dessa forma, como alimento habitualmente consumido, ${ }^{6}$ além de apresentar no mínimo $15 \%$ de citação, seja como item alimentar consumido isoladamente ou sendo ingredientes de preparações. ${ }^{17}$

Os alimentos do QFA que apresentaram menos de 15\% de citação e/ou consumo foram excluídos da lista final. Além disso, durante o processo de reestruturação do QFA, para os itens que apresentavam grandes variedades estabeleceu-se a denominação "outros", para identificar nomenclatura de alimentos regionais. Também foram incluídos, na lista do QFA, alimentos fontes de ferro e cálcio obtidos na Tabela Brasileira de Composição de Alimentos (TACO), por serem considerados intervenientes no metabolismo do chumbo, objeto de estudo da pesquisa matriz.

Após inclusão e exclusão de itens na lista final do QFA, os alimentos foram reagrupados, considerando-se a similaridade nutricional, e realizou-se a avaliação por juízes. Foram considerados juízes dois nutricionistas professores da área de Técnica Dietética e Alimentos, com experiência na classificação botânica e nutricional dos alimentos, bem como na elaboração e aplicação de questionários de frequência alimentar. 
Os pesquisadores avaliaram o QFA separadamente, quanto à composição dos grupos alimentares e à estrutura geral. Esse instrumento é do tipo qualitativo, pois dispõe da opção do consumo dos alimentos por grupos alimentares, sem a adição do tamanho da porção.

\section{Coleta e processamento dos dados}

A coleta de dados foi obtida a partir da aplicação do QFA inicial e R24h, conforme metodologia proposta por Slater, ${ }^{9}$ com a finalidade de identificar alimentos que possivelmente seriam de consumo habitual.

Os dados sobre o consumo alimentar foram digitados e analisados no software estatístico SPSS® versão 17.0. Para tanto, converteu-se a frequência de consumo de cada alimento em frequência diária, como preconizado por Coelho et al., ${ }^{4}$ cujo valor 1 representa o consumo de uma vez ao dia, e caso o alimento fosse consumido mais de uma vez ao dia, seria multiplicado pelo intervalo da frequência diária relatada. Para as opções que incluíram intervalos de tempo, utilizou-se a média do intervalo das frequências, dividida pelo período, se semanal (7) e mensal (30), conforme descrito no quadro $1 .{ }^{4}$ Assim, todas as frequências citadas foram transformadas em frequência diária, com a finalidade de utilizar apenas uma unidade temporal para realização das análises subsequentes, com finalidade de validação.

Quadro 1. Conversão de frequência do Questionário de Frequência Alimentar Diária. Santo Amaro-BA. Agosto a setembro, 2013.

\begin{tabular}{|lc|}
\hline \multicolumn{1}{|c|}{ Frequência } & Valor Atribuído \\
\hline Nunca & 0 \\
Menos de 1 vez mês & 0,03 \\
1 a 3 vezes mês & 0,07 \\
1 vez semana & 0,14 \\
2 a 4 vezes semana & 0,43 \\
1vez ao dia & 1 \\
2 vezes ou mais ao dia & 2 \\
\hline
\end{tabular}




\section{Resultados}

Dos 50 indivíduos analisados, $82 \%$ eram do sexo feminino, com média de idade de 39 anos (DP: 15).

Considerando os critérios de exclusão elucidados na metodologia, foram excluídos 38 itens alimentares do QFA inicial, representando 41,77\% dos alimentos da lista (quadro 2). Apesar da exclusão desses alimentos, não houve exclusão de nenhum dos grupos alimentares previamente selecionados.

Quadro 2. Alimentos excluídos da lista do QFA final. Santo Amaro-BA. Agosto a setembro, 2013.

\begin{tabular}{|c|c|}
\hline Grupo alimentar & Alimento excluído \\
\hline Frutas & $\begin{array}{l}\text { Cacau, caju, cana de açúcar, coco, graviola, melancia, } \\
\text { seriguela, tangerina }\end{array}$ \\
\hline Hortícolas & $\begin{array}{l}\text { Batata doce, beterraba, fruta pão, inhame, pepino, rúcula, } \\
\text { salsão, vagem }\end{array}$ \\
\hline Oleaginosas & Amendoim, castanhas \\
\hline Açúcares e açucarados & Mel, sobremesa, sorvete \\
\hline Laticínios & Requeijão \\
\hline Produtos de panificação & Salgados assados, salgados fritos, sanduíches \\
\hline Bebidas & Chás, suco artificial \\
\hline Carnes & Caprino, codorna, caça, ovinos \\
\hline Grãos & Feijão preto, feijão verde, feijão fradinho, andu \\
\hline Massas & Macarrão instantâneo \\
\hline Ovos & Ovo de codorna \\
\hline Farináceos & Tapioca \\
\hline
\end{tabular}

A lista final do QFA apresentou 53 itens alimentares, organizados por grupos de alimentos com características nutricionais e botânicas similares (quatro 3). 
Quadro 3. Alimentos que compõem o QFA final categorizados por grupo alimentar. Santo Amaro-BA. Agosto a setembro, 2013.

Grupos Alimentares

Frutas

Hortícolas

\section{Alimentos}

Abacaxi, acerola, banana, goiaba, laranja, limão, manga, maracujá.

Abobora, aipim, alface, batata inglesa, cebolinha, cenoura, chuchu, coentro, hortelã miúdo e grosso, pimenta, pimentão, quiabo, tomate

\begin{tabular}{ll}
\hline Oleaginosas & Azeite de dendê \\
\hline Açucares e açucarados & Bolo, açúcar \\
\hline Preparações regionais & Abará, acarajé \\
\hline Laticínios & Queijos, leite in natura \\
\hline Produtos de panificação & Pão \\
\hline Preparação & Sopa \\
\hline Bebidas & Agua, café, suco natural \\
\hline Carnes & Carne, frango, suíno, carnes conservadas no sal (carne \\
\hline Pescados & seca, carne do sol, charque, jabá) \\
\hline Grãos & $\begin{array}{l}\text { Peixe de água doce, peixe de água salgada, ostras, sururu, } \\
\text { chumbinho, siri, caranguejo, lambreta, mapé, sardinha }\end{array}$ \\
\hline Massas & Arroz, feijão carioca \\
\hline Ovos & Massas (lasanha, macarrão) \\
\hline Farináceos & Ovo de galinha \\
\hline
\end{tabular}

Os alimentos citados por no mínimo $15 \%$ da população estudada estão descritos na tabela 1, com destaque para alguns itens alimentares do grupo das frutas, que apresentaram percentual de consumo maior ou igual a 90\% (acerola, banana, goiaba, laranja e manga), grupo das hortícolas (abóbora, cenoura, coentro, pimentão, quiabo e tomate), grupo dos açúcares e açucarados (bolo, açúcar), laticínios (leite), produtos de panificação (pão), bebidas (água, suco natural), carnes (bovina, frango), grãos (arroz, feijão carioca), massas e farináceos. Os alimentos menos consumidos pela população com percentual de consumo menor que $60 \%$ foram os suínos, pescados e a preparação regional abará e pimenta. 
Tabela 1. Percentual dos alimentos consumidos pela população do estudo segundo a avaliação pelo método QFA. Santo Amaro-BA. Agosto a setembro, 2013.

\begin{tabular}{|c|c|c|}
\hline ITENS ALIMENTARES & $\mathrm{N}$ & $\%$ \\
\hline Abacaxi & 34 & 68 \\
\hline Acerola & 47 & 94 \\
\hline Banana & 45 & 90 \\
\hline Goiaba & 47 & 94 \\
\hline Laranja & 48 & 96 \\
\hline Limão & 42 & 84 \\
\hline Manga & 46 & 92 \\
\hline Maracujá & 41 & 82 \\
\hline Abobora & 46 & 92 \\
\hline Aipim & 42 & 84 \\
\hline Alface & 42 & 84 \\
\hline Batata inglesa & 41 & 82 \\
\hline Cebolinha & 34 & 68 \\
\hline Cenoura & 45 & 90 \\
\hline Chuchu & 33 & 66 \\
\hline Coentro & 48 & 96 \\
\hline Hortelã miúdo e grosso & 42 & 84 \\
\hline Pimenta & 26 & 52 \\
\hline Quiabo & 47 & 94 \\
\hline Pimentão & 45 & 90 \\
\hline Tomate & 47 & 94 \\
\hline Azeite de dendê & 41 & 82 \\
\hline Bolo & 47 & 94 \\
\hline Açúcar & 47 & 94 \\
\hline Abará & 30 & 60 \\
\hline
\end{tabular}




\begin{tabular}{lll}
\hline \multicolumn{1}{c}{ ITENS ALIMENTARES } & $\mathrm{N}$ & $\%$ \\
\hline Acarajé & 34 & 68 \\
Queijos & 38 & 76 \\
Leite in natura & 48 & 96 \\
Pão & 49 & 98 \\
Sopa & 41 & 82 \\
Carne bovina & 48 & 96 \\
Frango & 49 & 98 \\
Suínos & 23 & 46 \\
Carnes conservadas no sal & 44 & 88 \\
Pescados & 28 & 55 \\
Arroz & 49 & 98 \\
Feijão Carioca & 48 & 98 \\
Massas (lasanha, macarrão) & 47 & 94 \\
Ovo de galinha & 42 & 84 \\
Farinha de mandioca & 49 & 94 \\
Fubá de milho & 49 & 98 \\
Água & 49 & 94 \\
Café & 49 & 94 \\
\hline
\end{tabular}

Com auxílio do R24h, foram identificados dois alimentos (marisco mapé e sardinha) que inicialmente não tinham sido considerados na lista do QFA inicial. No entanto, considerandose o critério "frequência de consumo de pelo menos uma vez por semana" e de apresentar no mínimo $15 \%$ de citação, foram incluídos esses dois itens, visando abranger os hábitos alimentares da população.

Também foram inseridos alimentos fontes de cálcio e ferro, como carnes e laticínios, para atender à meta do estudo, de identificar alimentos fontes de cálcio e ferro consumidos pela população, a fim de avaliar sua interferência na absorção do chumbo pelo organismo. 


\section{Discussão}

O presente estudo permitiu descrever a elaboração de um QFA com 53 itens alimentares específicos para a população de uma região do Nordeste do Brasil. Destaca-se que com o instrumento elaborado e validado na população de estudo, a avaliação do consumo alimentar terá maior fidedignidade, por se aproximar da realidade local e dos hábitos alimentares, contribuindo com as pesquisas que investigam a relação entre consumo alimentar e condições de saúde.

Salienta-se que a mensuração da ingestão alimentar habitual é considerada uma das tarefas mais desafiadoras da epidemiologia nutricional. ${ }^{18}$ No entanto, diversas formas têm sido empregadas ao longo do tempo para facilitar e se aproximar do real consumo alimentar da população. Dentre estas, destacam-se a elaboração e validação de instrumentos para investigação de consumo alimentar da população.

Segundo Machado et al., ${ }^{19}$ o QFA é um instrumento importante para avaliar o consumo alimentar de determinada população, de modo mais prático e reduzido. Para a elaboração do QFA, é preciso determinar quais alimentos serão incluídos na lista, o tipo de classificação do QFA, se qualitativo, semi ou quantitativo e em seguida, decidir quais categorias de frequência de consumo serão empregadas. ${ }^{20}$

Com relação à construção da lista, Willett ${ }^{21}$ descreve uma abordagem metodológica a ser seguida, identificando os alimentos que são importantes fontes de nutrientes, usando informações a partir de tabelas de composição de alimentos, ou utilizando dados recolhidos por registro alimentar ou R24h. Assim, o estudo seguiu os procedimentos metodológicos adequados para construção da lista de alimentos do QFA, além de identificar os alimentos fontes de ferro e cálcio, para associá-los com o metabolismo de chumbo, objeto da pesquisa matriz. A vantagem de usar esta abordagem é que o número de itens na lista de alimentos é menor, tornando o QFA mais rápido e menos dispendioso na prática, e mais improvável de fadiga.

A aplicação de QFA por entrevistadores treinados diminui a frequência de erros no preenchimento do instrumento, mesmo com participantes de escolaridade de nível médio ou superior. ${ }^{22}$ Carvalho et al. ${ }^{23}$ reforçam a ideia de que a realização de um estudo-piloto permite o aprimoramento da equipe de coleta, bem como a reformulação das instruções. Reduz, desta forma, possíveis vieses que possam interferir na fidedignidade dos dados, contribuindo com a padronização do instrumento de coleta.

Destaca-se a importância do estudo-piloto para redução da lista de alimentos, o qual é geralmente realizado no mesmo grupo populacional em estudo. ${ }^{24}$ Apesar da simplificação da lista de alimentos que compõe o QFA, este não pode perder a essência, que é avaliar o mais próximo do real o consumo alimentar da população. Para tanto, deve-se atentar para as recomendações de 
autores como Fisberg et al. ${ }^{25}$ e Coluccii. ${ }^{20}$ Estes revelam que listas com menos de 50 itens alimentares não avaliam exatamente a ingestão alimentar, e que listas com mais de 100 itens podem causar fadiga e tédio durante o preenchimento, ocasionando vieses. Nesse sentido, observa-se que o QFA elaborado no presente estudo segue as recomendações descritas na literatura científica.

Em estudo sobre o desenvolvimento da lista de alimentos do QFA realizado por FurlanViebig \& Pastor Valero, ${ }^{26}$ foram utilizadas informações coletadas pelos R24h, em que os indivíduos relataram detalhadamente os alimentos e bebidas consumidos nas 24 horas anteriores à entrevista; estes alimentos serviram para compor a lista do QFA do estudo.

A redução da extensão da lista de alimentos do QFA é importante como forma de simplificar, facilitar a compreensão e aplicação do questionário em campo, evitando recusas. No entanto, a este procedimento deve ser realizado de forma metodologicamente cuidadosa, com a finalidade de manter na lista final os principais alimentos que representam o consumo habitual dos indivíduos e/ou populações..$^{23}$

De modo geral, estudos que objetivam reduzir listas de alimentos de questionários de frequência são desenvolvidos para avaliação do consumo de nutrientes específicos ou grupo de alimentos.

Considerando a importância da lista de alimentos para um melhor resultado sobre o estudo dos hábitos alimentares, o QFA inicial do presente estudo foi uma ferramenta essencial para a elaboração do QFA final (modelo reduzido). É resumido em 53 alimentos agrupados em 15 grupos alimentares, representando aproveitamento de 58,24\% dos itens alimentares presentes no QFA inicial. Esse percentual está próximo ao obtido por Chiara et al., ${ }^{27}$ que foi de $50 \%$ de aproveitamento dos alimentos do QFA inicial para o QFA final em estudo com adolescentes. Tal fato reforça a necessidade de reduzir os itens na lista de alimentos com públicos diversos.

Nessa perspectiva, observa-se que a lista de alimentos que compõem o QFA é representativa da população em estudo, por levar em consideração, no momento da elaboração, condicionantes importantes da avaliação do consumo alimentar, a exemplo de acesso, renda, cultura, regionalidade e composição nutricional dos alimentos, considerações estas descritas pelo Guia Alimentar Brasileiro. ${ }^{10}$

A lista do QFA deste estudo foi comparada às informações contidas na $\mathrm{POF},{ }^{16}$ com o objetivo de detectar alimentos que não tivessem sido relatados nos R24h, mas que poderiam ser componentes importantes para a ingestão habitual da população estudada. No entanto, observou-se que os alimentos adquiridos pelas famílias de Santo Amaro encontravam-se incluídos na lista do QFA final, revelando precisão na coleta dessas informações, pois se aproximaram da dieta habitual.

Segundo Matarazzo, ${ }^{28}$ a qualidade das informações sobre a dieta será determinada pela precisão e pela validade do instrumento de registro, que deve ser avaliado especificamente para cada população em estudo. 
Salienta-se que tanto o QFA e o R24h apresentam limitações inerentes do próprio método, a exemplo do viés de memória, ou seja, o indivíduo pode ter dificuldade em recordar todos os alimentos consumidos. No entanto, foram empregados procedimentos descritos na literatura que minimizam esses erros e contribuem com a memória do entrevistado, como por exemplo, técnicas de recordação (listagem rápida, descrição detalhada e revisão). ${ }^{25}$ Assim, todos os cuidados para minimização de erros foram considerados na elaboração do instrumento, de forma que este, uma vez aplicado, possa representar com maior fidedignidade o consumo alimentar da população em estudo.

Uma limitação do estudo proposto consiste em número amostral pequeno; no entanto observouse, na literatura científica, que outros estudos que trabalham com desenvolvimento e validação de QFA utilizam esse mesmo número de amostra, além de que este número é referenciado por Slater et al. ${ }^{9}$ como borderline para execução.

Quanto aos aspectos positivos da pesquisa, destaca-se que foi desenvolvida em uma população do Nordeste do Brasil, carente de estudos que envolvem a área de consumo alimentar. Além disso, para elaboração do QFA, foram levados em consideração todos os pressupostos necessários para análise. Ressalta-se, no entanto, que foram utilizados apenas um QFA e um R24h, e para maior acurácia em futuras análises recomenda-se mais de uma aplicação de R24h, visando melhor precisar a investigação do consumo alimentar da população.

\section{Conclusão}

Com base nos dados observados, conclui-se que o QFA inicial e o R24h foram instrumentos importantes que delinearam metodologicamente a construção do QFA proposto, sobretudo no que se refereàa redução da lista de alimentos, expressando maior qualidade do instrumento, praticidade e otimização do tempo de aplicação.

É possível que a lista de 53 alimentos do presente QFA represente a dieta "habitual" da população de Santo Amaro-BA, no Nordeste do Brasil. Quando comprovada a validade do instrumento (estudo em desenvolvimento), ele poderá ser utilizado em futuros estudos epidemiológicos que busquem investigar a relação entre dieta e saúde.

Estudos como este são relevantes por descreverem metodologicamente a construção de um instrumento de investigação de consumo alimentar específico para a população, aproximando-se da intrínseca relação entre consumo alimentar real e consumo alimentar observado e contribuindo com outros estudos que tenham o mesmo objetivo. Além disso, os resultados da avaliação do consumo alimentar investigado por esses instrumentos podem ser associados às condições de saúde da população, no intuito de identificar grupos alimentares protetores e grupos alimentares de risco para determinado problema de saúde, contribuindo com as políticas de saúde pública. 


\section{Referências}

1. Monteiro CA. Nutrition and health. The issue is not food, nor nutrients, so much as processing. Public Health Nutrition 2009: 12(5):729-731.

2. Neumann AICP, Martins IS, Marcopito LF, Araújo EAC. Padrões alimentares associados a fatores de risco para doenças cardiovasculares entre residentes de um município brasileiro. Rev. Panam. Salud Publica 2007; 22(5):329-339.

3. World Health Organization. Diet, nutrition and the prevention of chronic diseases. Geneva: WHO; 2003.

4. Coelho NLP, Cunha DB, Esteves APP, Lacerda EMA, Theme Filha MM. Padrão de consumo alimentar gestacional e peso ao nascer. Rev. Saúde Pública 2015; 49(62):2-10.

5. Crozier SR, Robinson SM, Godfrey KM, Cooper C, Inskip HM. Women's dietary patterns change little from before to during pregnancy. J. Nutr. 2009; 139(10):1956-63.

6. Willet WC. Nutritional epidemiology. 20 ed. New York: Oxford University; 1998.

7. Fisberg RM, Marchioni DML, Colucci ACA. Avaliação do consumo alimentar e da ingestão de nutrientes na prática clínica. Arq. Bras. Endocrinol. Metab. 2009; 617-624.

8. Voci SM, Enes CC, Slater B. Validação do Questionário de Frequência Alimentar para Adolescentes (QFAA) por grupos de alimentos em uma população de escolares. Rev. Bras. Epidemiol. 2008; 11(4):561-572.

9. Slater B, Philippi ST, Marchioni DM, Fisberg RM. Validação de Questionário de Frequência Alimentar - QFA: considerações metodológicas. Rev. Bras. Epidemiol. 2003: 6(3):200-208.

10. Brasil. Ministério da Saúde. Secretaria de Atenção à Saúde. Coordenação-Geral da Política de Alimentação e Nutrição. Guia alimentar para a população brasileira: versão para consulta pública. Brasília: Ministério da Saúde; 2014. 91 p.

11. Cade J, Thompson R, Burley V, Warm D. Development, validation and utilization of food frequency questionnaires: a review. Public Health Nutr. 2002; 5(4):567-87.

12. Burley V, Cade J. Consensus document on the development, validation and utilization of food frequency questionnaires. The Fourth International Conference on Dietary Assessment Methods; 17-20 sept. 2000; Tucson, Arizona (USA).

13. Brasil. Resolução n ${ }^{\circ} 466$, de 12 de dezembro de 2012. Aprova normas regulamentadoras de pesquisas envolvendo seres humanos Diário Oficial da União 13 jun. 2013; Seção I, p. 59-62.

14. Instituto Brasileiro de Geografia e Estatística. Produção Agrícola Municipal. [acesso em 06 abr. 2014]. Disponível em: http://www.ibge.gov.br/home/estatistica/pesquisas/pesquisa_resultados. php?id_pesquisa $=44$

15. Brasil. Ministério da Saúde. Alimentos regionais brasileiros. Brasília: Ministério da Saúde; 2002.

16. Instituto Brasileiro de Geografia e Estatística. Pesquisa de orçamentos familiares 2008-2009: análise do consumo alimentar pessoal no Brasil. Rio de Janeiro: IBGE; 2011. 150 p. 
17. Ferreira MG, Silva NF, Schmidt FD, Silva RMVG, Sichieri R, Guimarães LV, et al. Desenvolvimento de questionário de frequência alimentar para adultos em amostra de base populacional de Cuiabá, Região Centro-Oeste do Brasil. Rev. Bras. Epidemiol. 2010; 13(3):413-424.

18. Silva TAV, Lima SM. Procedimentos metodológicos empregados em questionários de frequência alimentar elaborados no Brasil: uma revisão sistemática. Rev. Nutr. 2012; 25(6):785-797.

19. Machado FCS, Ruth LH, Olinto MTA, Anjos LA, Wahrlich V, Waissmann W. Reprodutibilidade e validade de um questionário de frequência alimentar por grupos de alimentos, em adultos da Região Metropolitana de Porto Alegre, Brasil. Rev. Nutrição 2012; 25(1):65-77.

20. Colucci ACA, Slater B, Philippi ST. Etapas para desenvolvimento de um questionário de frequência alimentar. Rev. Bras. Ciências da Saúde 2005; III(6):7-12.

21. Willet WC. Food frequency methods. $2^{\circ}$ ed. New York: Oxford University; 1990.

22. Cardoso MA, Stocco PR. Desenvolvimento de um questionário quantitativo de frequência alimentar em imigrantes japoneses e seus descendentes residentes em São Paulo, Brasil. Cad. Saúde Publica 2000; 16(1):107-114.

23. Carvalho FS, Laer NMV, Sachs A, Salvo VLMA, Coelho LC, Santos GMS, et al. Desenvolvimento e pré-teste de um questionário de frequência alimentar para graduandos. Rev. Nutr. 2010; 23(5):847-857.

24. Villar BS. Desenvolvimento e validação de um questionário semi-quantitativo de frequência alimentar para adolescentes. [tese]. [São Paulo]: Universidade de São Paulo, Faculdade de Saúde Pública; 2001.

25. Fisberg R, Slater B, Marchioni D, Martini L. Inquéritos alimentares, métodos e bases científicos. Barueri: Manoele; 2005.

26. Furlan-Viebig R, Pastor-Valero M. Development of a food frequency questionnaire to study diet and non-communicable diseases in adult population. Rev. Saúde Pública 2004; 38(4):581-584.

27. Chiara VL, Barros ME, Costa LP, Martins PD. Redução de lista de alimentos para questionário de frequência alimentar: questões metodológicas na construção. Rev. Bras. Epidemiol. 2007; 10(3):410-420.

28. Matarazzo HCZ, Marchioni DML, Figueiredo RAO, Slater B, Eluf Neto J, Wünsch Filho V. Reprodutibilidade e validade do questionário de frequência de consumo alimentar utilizado em estudo caso-controle de câncer oral. Rev. Bras. Epidemiol. 2006; 9(3):316-324. 
